\title{
Structural damage identification and location using grammian matrices
}

\author{
D.D. Bueno ${ }^{\mathrm{a}}$, C.R. Marqui ${ }^{\mathrm{a}}$, V. Lopes Jr. ${ }^{\mathrm{a}, *}$, M.J. Brennan ${ }^{\mathrm{a}}$ and Daniel J. Inman ${ }^{\mathrm{b}}$ \\ ${ }^{a}$ UNESP - Univ Estadual Paulista, Faculdade de Engenharia de Ilha Solteira, Grupo de Materiais e Sistemas \\ Inteligentes - GMSINT, Av. Brasil, n. 56, Centro, Ilha Solteira, SP, Brazil \\ ${ }^{\mathrm{b}}$ Center for Intelligent Material Systems and Structures, Virginia Tech, Blacksburg, VA, USA
}

Received 9 December 2010

\begin{abstract}
In this paper, an approach using observability and controllability grammian matrices is proposed to determine if structural damage has occurred together with an estimate of its location. The theory is outlined and simulations are carried out on a simple structure to demonstrate the method. Experimental tests were also carried out to demonstrate the validity of the approach using real signals. The dynamic properties of the structure are identified using the eigensystem realization algorithm (ERA) and a reduced order state-space model of the system subsequently constructed. Either the observability or controllability grammians can then be used depending on the number of sensors available. It is shown that these are sensitive to both the degree and location of the damage and offer promise for structural health monitoring applications.
\end{abstract}

\section{Introduction}

One of the desirable properties of a Structural Health Monitoring (SHM) system is the ability to detect and interpret adverse changes in a structure in order to improve reliability and reduce life-cycle costs. The challenge in designing a SHM system is knowing what changes to look for and how to identify them. Different approaches for SHM have been proposed for damage location. Most of the methods take advantage of the dynamical behaviour of the structure [7]. There is no acknowledged best technique for general applications. Methods that have been developed so far tend to be case dependent.

Aerospace structures have one of the highest payoffs for SHM applications since damage can lead to catastrophic and expensive failures, and the vehicles involved undergo regular costly inspections. Currently $27 \%$ of the life cycle cost of an average aircraft, both for commercial and military vehicles, is spent on inspection and repair; and it excludes the opportunity cost associated with the time the aircraft is grounded for scheduled maintenance [11]. Structural Health Monitoring systems are attracting attention for controlling or reducing risks associated with civil and mechanical structures due to natural hazards such as large earthquakes [22]. This trend has been accelerated after the 1994 Northridge Earthquake and the 1995 Hyogo-Ken Nanbu Earthquake.

In general, a considerable number of sensors are necessary for damage location with some precision [21]. However, in practical situations, the number of sensors is generally limited. Sensors are used to record variables such as strain, acceleration, sound waves, electrical or magnetic impedance, pressure or temperature. It has been estimated that a SHM system for an aerospace vehicle would require between 100 and 1000 sensors, depending on its size and the desired coverage area [20].

In this context, this paper proposes a novel approach using observability and controllability grammian matrices. The concept of observability and controllability was introduced by Kalman [16], and the main applications of grammian matrices since then have been for control design and optimal placement of actuators in smart structures [2,

\footnotetext{
*Corresponding author. E-mail: vicente@ dem.feis.unesp.br.
} 
17,24]. An approximate approach that uses the size of the grammian matrices was applied to determine the optimal actuator location for a vibrating system [1]. A combination of the geometric mean and the determinant of the grammian matrix have been used as a different measure to evaluate the sensor and actuator locations [10]. Also, a method that maximizes the energy dissipation was proposed to find an optimal set of sensor/actuator positions and the feedback gains [25]. The orthogonal projection of structural modes onto the intersection subspace of the controllable and observable subspaces corresponding to an actuator/sensor pair was used as a criterion to find the optimal placement of sensors and actuators for a large structure [18].

Many methodologies have been proposed for structural health monitoring. Van der Auweraer and Peeters [28] review many international research programs and activities, which are devoted to this topic. Sohn et al. [27] report new technical developments published between 1996 and 2001 in SHM. An interesting viewpoint on a system's remaining useful life was addressed in a report from Los Alamos National Laboratory [6]. The authors foresee that damage prognosis will be an important engineering research topic in the near future because of the potential life-safety and economic advantages that this technology can provide.

Gawronski and Sawicki [9] proposed a non-destructive technique to determine damage location using the $\mathrm{H}_{2}$ modal norm. The authors presented a numerical example of a truss structure where two cases of damage were considered by a reduction in stiffness. Deraemaeker and Preumont [4] used a large network of sensors to which a programmable linear combiner was attached. The linear combiner was programmed to work as a modal filter. The frequency content of the output of the modal filter was proposed as a feature for damage detection. It was shown that if local damage is present, spurious peaks appear in the frequency response function (FRF) of the modal filter whereas if temperature changes are considered, the FRF of the modal filter is shifted but its shape remains unchanged. Silva et al. [26] describe the application of an SHM technique based on electrical measurements obtained by piezoceramics (PZT) patches bonded to lightweight structures. They used an approach based on time-series, auto-regressive moving average with the input being ambient vibration (exogenous) (ARMAX), and statistical process control (SPC) charts for linear prediction to detect and locate damage.

This paper presents numerical simulations and experimental validation for two approaches to damage detection and location using grammian matrices. The rationale for using this approach is that these grammians contain, in a single index, information on the dynamics of the structure and on the excitation/measurement of the vibration. Because of this the grammians will be sensitive to damage as it changes the dynamic behaviour of the system. The first method, proposed by Bueno et al. [3], is based on observability grammian matrices, and the second method is based on controllability grammian matrices. For cases where the number of sensors is small, the second technique is more appropriate. The approach presented here allows the detection and location of damage using a simple algorithm and can be easily implemented. The feasibility of the method is demonstrated by experimental application on a cantilever beam.

Part of the procedure to determine the observability and controllability grammian matrices requires an identification method for a state-space model of the structrure. There are different identification methods proposed in the literature, as for instance, the parameter identification method by polynomial functions [5,23]; complex exponential [19]. In this paper, however, the eigensystem realization algorithm (ERA) $[13,14]$ is used and this is briefly described in Section 2. Following this, the way in which the observability and controllability grammians are formed and used to detect and locate damage is described. Some simulations are presented and then the experimental work to validate the approach is described before the paper is closed with some general conclusions.

\section{Procedure}

The approach to identify damage using grammians is to measure the dynamic behavior of the healthy structure, and then to periodically check how this changes with time. Figure 1 depicts the processes that the measured signals undergo to determine the state-space model of the structural dynamics and to check whether damage has occurred and its location. The acceleration signals are first passed through a low-pass filter and then the eigensystem realization algorithm (ERA) $[13,14]$ is applied to determine the system parameters. Once the state-space model has been determined the observability and controllability grammians can be calculated and the health of the structure determined. 


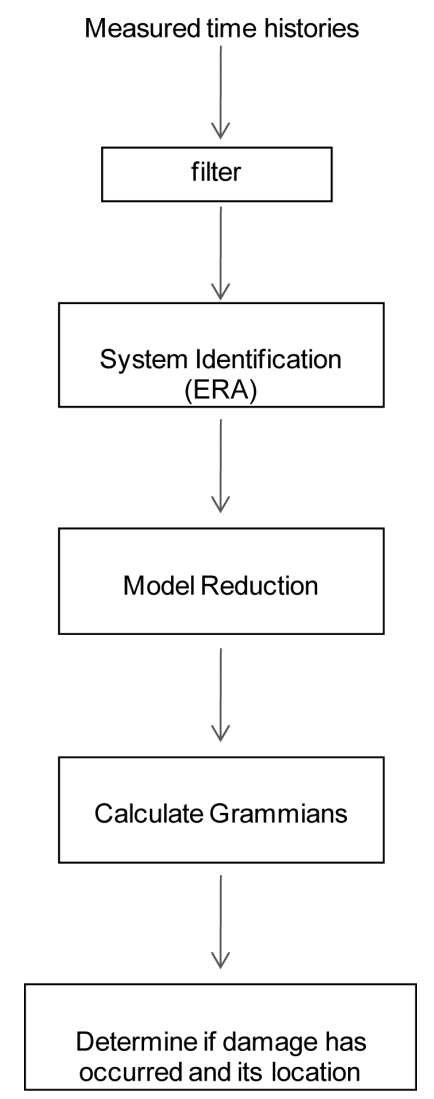

Fig. 1. Diagram showing the steps in characterizing damage.

\subsection{Experimental identification of the structural matrices}

The state-space model in continuous times can be described from a finite element model. The procedure for doing this is described in the Appendix. The discrete time state-space model is given by

$$
\begin{aligned}
& \mathbf{x}(k+1)=\mathbf{A}_{d} \mathbf{x}(k)+\mathbf{B}_{d} \mathbf{u}(k) \\
& \mathbf{y}(k)=\mathbf{C}_{d} \mathbf{x}(k)
\end{aligned}
$$

where $\mathbf{x}(k), \mathbf{y}(k)$ and $\mathbf{u}(k)$ are respectively the state, output and input vectors and $\mathbf{A}_{d}, \mathbf{B}_{d}, \mathbf{C}_{d}$ are the state, input and output matrices of the discrete state-space model respectively. In the example presented in this paper the excitation is by an instrumented hammer and the output vector for one of the measurement points is a filtered acceleration signal given by vector $\mathbf{y}=[y(0), y(1), y(2), \ldots y(N)]$. This is used to form the Hankel matrix given by

$$
\mathbf{H}(k-1)=\left[\begin{array}{cccc}
\mathbf{y}(k) & \mathbf{y}(k+1) & \ldots & \mathbf{y}(k+j) \\
\mathbf{y}(k+1) & \mathbf{y}(k+2) & \ldots & \mathbf{y}(k+j+1) \\
\vdots & \vdots & \vdots & \vdots \\
\mathbf{y}(k+i) & \ldots & \ldots & \mathbf{y}(k+i+j)
\end{array}\right]
$$

where $i$ and $j$ are integers. The state, input and output matrices $\mathbf{A}_{d}, \mathbf{B}_{d}, \mathbf{C}_{d}$ respectively of the discrete system can be determined using matrices $\mathbf{H}(0)$ and $\mathbf{H}(1)$. The Hankel matrix is also known as the block data matrix [14]. Decomposition of $\mathbf{H}(0)$ into singular values yields the diagonal matrix $\mathbf{S}$, which contains the singular values of $\mathbf{H}(0)$ arranged in descending order, and the matrices $\mathbf{U}$ and $\mathbf{V}$ containing the left and right singular vectors respectively, so that

$$
\mathbf{H}(0)=\mathbf{U S V}^{T}
$$


Selecting the $p$ highest singular values, gives the matrix $\mathbf{S}_{p}$ which contains the $p$ highest singular values and the matrices of associated singular vectors $\mathbf{U}_{p}$ and $\mathbf{V}_{p}$ from which the state, input and output matrices $\mathbf{A}_{d}, \mathbf{B}_{d}, \mathbf{C}_{d}$ can be determined [14].

$$
\begin{aligned}
\mathbf{A}_{d} & =\mathbf{S}_{p}^{-1 / 2} \mathbf{U}_{p}^{T} \mathbf{H}(1) \mathbf{V}_{p} \mathbf{S}_{p}^{-1 / 2} \\
\mathbf{B}_{d} & =\mathbf{S}_{p}^{1 / 2} \mathbf{V}_{p}^{T} \\
\mathbf{C}_{d} & =\mathbf{U}_{p}^{1 / 2} \mathbf{S}_{p}^{T}
\end{aligned}
$$

Once the matrices $\mathbf{A}_{d}, \mathbf{B}_{d}, \mathbf{C}_{d}$ have been computed, the continuous state-space model with matrices $\mathbf{A}, \mathbf{B}, \mathbf{C}$ can be obtained using the discrete to continuous transformation.

\subsection{Damage detection and location using observability and controllability grammians}

Controllability and observability properties of a linear time-invariant system can be heuristically described as follows. The system dynamics described by the state variable $\mathbf{x}(t)$ is excited by the input $\mathbf{u}(t)$ and measured by the output $\mathbf{y}(t)$. However, the input may not be able to excite all states (or, equivalently, move it in an arbitrary direction), and not all states are represented at the output (or, equivalently, the system state cannot be recovered from a record of the output measurements). If the input excites all states, the system is controllable. If all the states are represented in the output, the system is observable.

The metrics, based on observability and controllability, use matrices $\mathbf{A}, \mathbf{B}$ and $\mathbf{C}$ simultaneously. Together, these matrices contain information about the dynamics of the structure, how it is observed at the sensor positions and how it is excited at the actuator positions. This information is therefore included in the metrics and so any changes in the structure due to damage is also reflected in the metrics. In this way, the proposed methodology can identify if there is damage or not and the position at which damage occurs within a single index.

\subsubsection{Observability grammian matrices}

Observability involves the system matrix $\mathbf{A}$ and the output matrix $\mathbf{C}$. A linear system is observable at $t_{0}$ if the state $\mathbf{x}\left(t_{0}\right)$ can be determined from the output $\mathbf{y}(t), t \in\left[t_{0}, t_{1}\right]$, where $t_{1}>t_{0}$ is some finite time. If this statement is true for all $t_{0}$ and all initial states $\mathbf{x}\left(t_{0}\right)$, the system is completely observable. Otherwise, the system is unobservable. There are many criteria to determine system observability [15,29]. A linear time-invariant system with $r$ outputs is completely observable if and only if the $r N \times N$ matrix $\left[\mathbf{C ~ C A ~} \mathbf{C A}^{2} \ldots \mathbf{C A}^{N-1}\right]^{T}$ has rank $N=\operatorname{size}(\mathbf{A})$. This criterion, although simple, has two serious drawbacks. First, it answers the observability question only in terms of yes or no. Second, it is useful only for a system of small dimension. Assume, for example, that the system is of dimension $N=100$. In order to answer the observability question, one has to find powers of $\mathbf{A}$ up to 99 . Finding $\mathbf{A}^{99}$ for a $100 \times 100$ matrix is a numerical task that easily results in numerical overflow.

An alternative approach uses grammians to determine the system properties. Grammians express the observability properties qualitatively and avoid numerical difficulties. The observability grammian is defined as [15].

$$
\mathbf{W}_{\mathbf{o}}(t)=\int_{0}^{t} \exp \left(\mathbf{A}^{T} t\right) \mathbf{C}^{T} \mathbf{C} \exp (\mathbf{A} t) d t
$$

where the subscript $\mathrm{o}$ stands for observability. Alternatively, it can be determined from the following system of differential equations

$$
\dot{\mathbf{W}}_{\mathbf{o}}(t)=\mathbf{A}^{T} \mathbf{W}_{\mathbf{o}}+\mathbf{W}_{\mathbf{o}} \mathbf{A}+\mathbf{C}^{T} \mathbf{C}
$$

For a stable system, the stationary solutions of the above equations are obtained by assuming $\dot{\mathbf{W}}_{\mathbf{O}}=\mathbf{0}$. In this case, the grammian vector is determined from the Lyapunov equation

$$
\mathbf{A}^{T} \mathbf{W}_{\mathbf{O}}+\mathbf{W}_{\mathbf{O}} \mathbf{A}+\mathbf{C}^{T} \mathbf{C}=\mathbf{0}
$$

For a stable $\mathbf{A}$, the grammian $\mathbf{W}_{\mathbf{O}}$ is positive definite. Note that matrix $\mathbf{C}$ is only related to the position of the $j$-th sensor, and that Eq. (9) has to be formed for each sensor individually. Denoting the $j$-th sensor observability matrix 
of the healthy structure by $\left\|W_{\mathrm{oh}, j}\right\|=\operatorname{trace}\left(\mathbf{W}_{\mathbf{O}}\right)$, and the $j$-th sensor observability matrix of the damaged structure by $\left\|W_{\mathrm{od}, j}\right\|$, where the subscripts $\mathrm{h}$ and $\mathrm{d}$ stand for healthy and damaged respectively. The $j$-th sensor index to characterize structural damage is defined as the normalized difference between the $j$-th sensor of the healthy and damaged structure. The sensor index reflects the impact of that specific structural damage on the $j$-th sensor

$$
\sigma_{\text {sensor }, j}=\frac{\left|\left\|W_{\mathrm{oh}, j}\right\|^{2}-\left\|W_{\mathrm{od}, j}\right\|^{2}\right|}{\left\|W_{\mathrm{oh}, j}\right\|^{2}}
$$

The results are normalized with the maximum value set to unity. Thus, if the states of the damaged system as measured by the $j$-th sensor are significantly different from the healthy structure the index $\sigma_{\text {sensor, } j} \rightarrow 1$; if the states are similar then $\sigma_{\text {sensor, } j} \rightarrow 0$.

\subsubsection{Controllability grammian matrices}

Controllability, as a coupling between the input and the states, involves the system matrix $\mathbf{A}$ and the input matrix B. A linear system is controllable at $t_{0}$ if it is possible to find a piecewise continuous input $\mathbf{u}(t), t \in\left[t_{0}, t_{1}\right]$, that will transfer the system from initial state, $\mathbf{x}\left(t_{0}\right)$, to an arbitrary state $\mathbf{x}\left(t_{1}\right)$, at finite $t_{1}>t_{0}$. If this is true for all initial $\mathbf{x}\left(t_{0}\right)$ the system is completely controllable. Otherwise, the system is uncontrollable. There are many criteria to determine system controllability [15,29]. A linear time-invariant system with $s$ inputs is completely controllable if and only if the $N \times s N$ matrix of $\left[\begin{array}{lllll}\mathbf{B} & \mathbf{A B} & \mathbf{A}^{2} \mathbf{B} & \ldots & \mathbf{A}^{N-1} \mathbf{B}\end{array}\right]$ has rank $N=\operatorname{size}(\mathbf{A})$. Similar to the observability approach, an alternative is to use the grammian. The controllability grammian is defined as [15]

$$
\mathbf{W}_{\mathbf{c}}(t)=\int_{0}^{t} \exp (\mathbf{A} t) \mathbf{B B}^{T} \exp \left(\mathbf{A}^{T} t\right) d t
$$

Alternatively, it can be determined from the following system of differential equations

$$
\dot{\mathbf{W}}_{\mathbf{c}}(t)=\mathbf{A W}_{\mathbf{c}}+\mathbf{W}_{\mathbf{c}} \mathbf{A}^{T}+\mathbf{B B}^{T}
$$

For a stable system, the stationary solutions of the above equations are obtained by assuming $\dot{\mathbf{W}}_{\mathbf{c}}=\mathbf{0}$. In this case, the grammian matrix is determined from the Lyapunov equation

$$
\mathbf{A W}_{\mathbf{c}}+\mathbf{W}_{\mathbf{c}} \mathbf{A}^{T}+\mathbf{B B}^{T}=\mathbf{0}
$$

For a stable $\mathbf{A}$, the grammian $\mathbf{W}_{c}$ is positive definite. Note that matrix $\mathbf{B}$ is only related to the position of the $j$-th actuator and that Eq. (13) has to be formed for each actuator separately. Denoting the $j$-th controllability input index of the healthy structure by $\left\|W_{\mathrm{ch}, j}\right\|=\operatorname{trace}\left(\mathbf{W}_{\mathbf{c}}\right)$ and the $j$-th controllability input index of the damaged structure by $\left\|W_{\mathrm{cd}, j}\right\|$. The $j$-th input index to characterize structural damage is defined as the normalized difference between the $j$-th input of the healthy and damaged structure. The input index reflects the impact of that specific structural damage on the $j$-th input position

$$
\sigma_{\text {input }, j}=\frac{\left|\left\|W_{\mathrm{ch}, j}\right\|^{2}-\left\|W_{\mathrm{cd}, j}\right\|^{2}\right|}{\left\|W_{\mathrm{ch}, j}\right\|^{2}}
$$

The results are also normalized with the maximum value set to unity. Thus, if the states of the damaged system are significantly different from the healthy structure when it is excited at the $j$-th position then $\sigma_{\text {input }, j} \rightarrow 1$; if the states are similar then $\sigma_{\text {input }, j} \rightarrow 0$.

\section{Simulations}

The effectiveness of the approach was determined through simulations on a steel cantilever beam, $420 \mathrm{~mm}$ long, $37 \mathrm{~mm}$ wide and $5 \mathrm{~mm}$ thick. It was discretised as shown in Fig. 2 with 10 elements and 11 structural nodes, resulting in 20 structural degrees-of-freedom (dofs) (two dofs per node). The finite element approach was used to model the beam and then a state-space model of the structure was developed using the procedure described in the Appendix. 


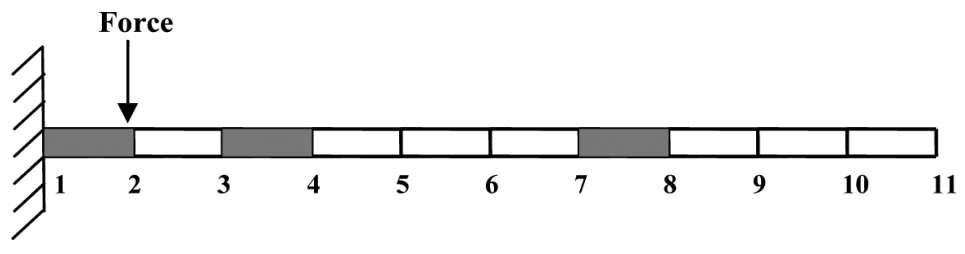

Damage Position

Fig. 2. Finite element model for the cantilever beam.

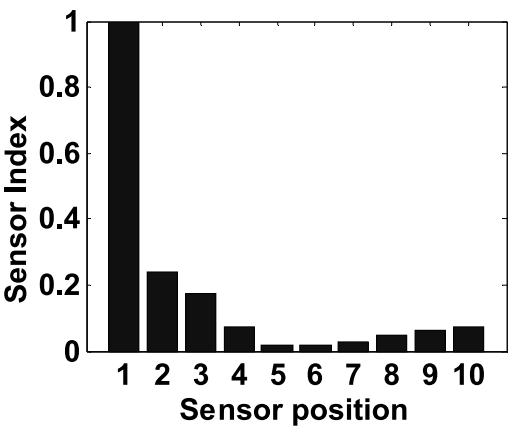

(a) Damage case 1 - stiffness reduction of $2 \%$ in element 1 .

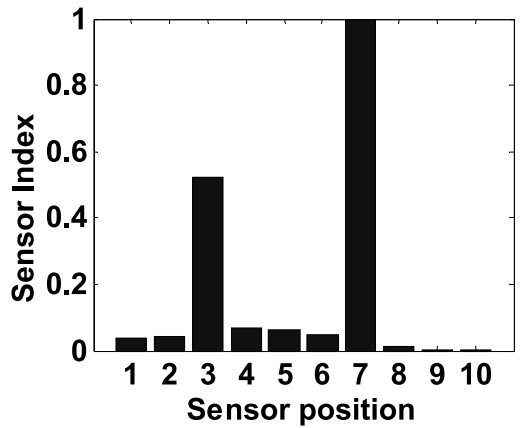

(b) Damage case 2 - stiffness reduction of $3 \%$ in element 3 and $5 \%$ in element 7 .

Fig. 3. Sensor index calculated using Eq. (10) for each of the sensor positions - simulations.

Subsequently the observability and controllability grammians were determined using the approach described in the previous section.

Two damage cases were considered. The first is a $2 \%$ reduction in the stiffness of element 1 and the second is a $3 \%$ and $5 \%$ reduction in the stiffness of elements 3 and 7 , respectively. Acceleration time histories of all the structural elements due to an out-of-plane impulsive force applied at node 2 are used to determine the health of the structure. Figures $3 \mathrm{a}$ and $3 \mathrm{~b}$ shows the sensors indexes calculated using the observability grammian for each of the 10 measurement positions for the first and second cases of damage respectively. In Fig. 3a, it can be clearly seen that the structural damage is located in element 1, and in Fig. $3 \mathrm{~b}$ the damage is clearly located in elements 3 and 7.

When considering the controllability grammian only one acceleration signal of node 11 is used and the out-ofplane impulsive force is applied at all the structural nodes. Figures $4 \mathrm{a}$ and $4 \mathrm{~b}$ show the input indexes for cases 1 and 2 , respectively. In the first case, when the force is applied next to the damage, it is easy to see the effect of damage on the input index. In the second case, when there are two damaged elements, the input indices show the damage regions. Using the controllability grammian does not give such a clear, distinct indication of where the damage is. This is probably because the damage in this case is in two positions and the effect of the damage is similar on all input positions. However, it could be a useful approach when it would not be feasible to instrument a structure with many sensors, but where it would be possible to excite the structure at many locations with an instrumented hammer, for example in truss structures or plates.

\section{Experimental work}

To validate the theoretical predictions some experimental work was conducted. Figure 5 shows the experimental setup to detect and locate damage using the observability grammian. The properties of the beam were the same as those used in the previous section. Tests were performed by exciting the structure with a PCB impact hammer, first on the healthy structure and then on the damaged structure. The output signals were measured with PCB type 352C22 accelerometers in the positions shown in Fig. 5 and were acquired using SignalCalc ACE II. A low-pass 


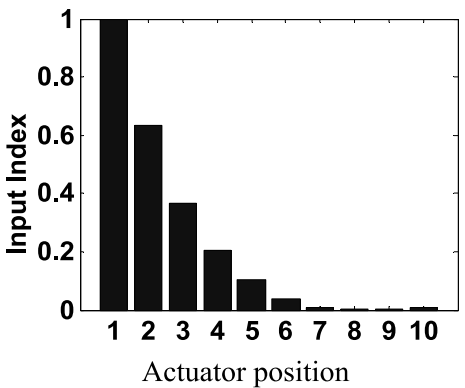

(a) Damage case 1 - stiffness reduction of $2 \%$ in element 1

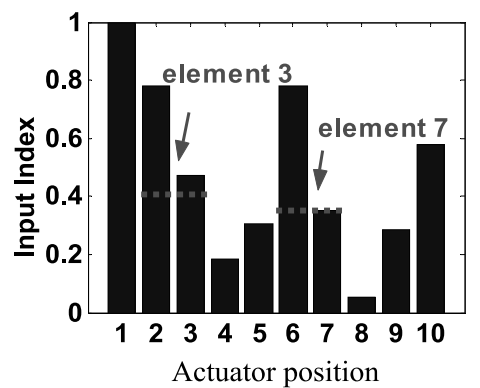

(b) Damage case 2 - stiffness reduction of $3 \%$ in element 3 and $5 \%$ in element 7

Fig. 4. Input index calculated using Eq. (14) for each of the actuator position.

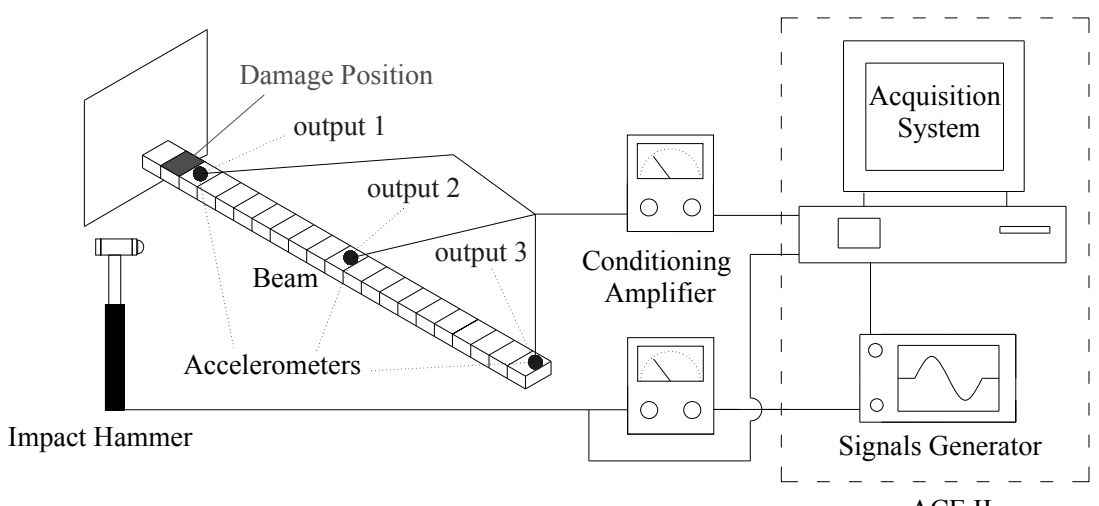

(a) schematic

ACE II

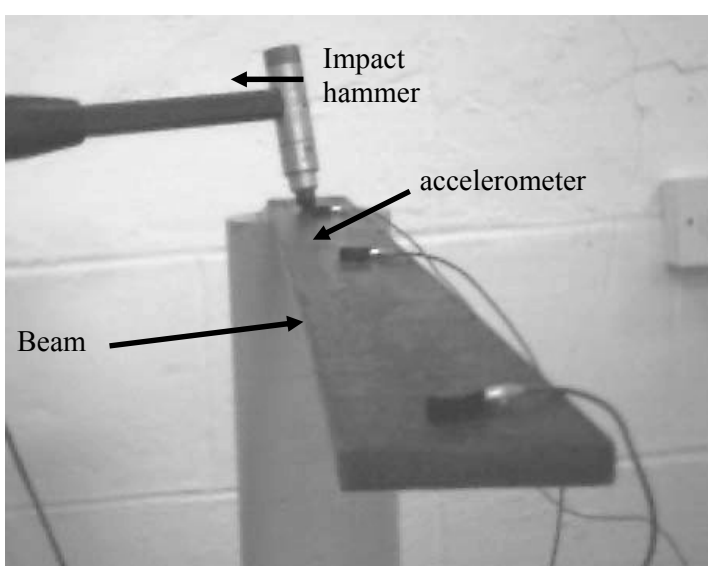

(b) photograph

Fig. 5. Experimental setup used for damage location using the observability grammian. (steel cantilever beam with $420 \mathrm{~mm}$ long, $37 \mathrm{~mm}$ wide and $5 \mathrm{~mm}$ thick).

filter with a cut-off frequency of $1 \mathrm{kHz}$ was used. The parameters of the system were then identified by using the Eigensystem Realization Algorithm (ERA) described in Section 2. The order of the system was chosen to be equal to six, so, only the first three modes were used to compute the grammian matrices. Damage was simulated by adding additional mass $(16 \mathrm{~g})$ at the damage location on the beam rather than reducing the stiffness as in the simulations.

Figures 6a-c show the frequency response functions before and after the damage was introduced. Although the 


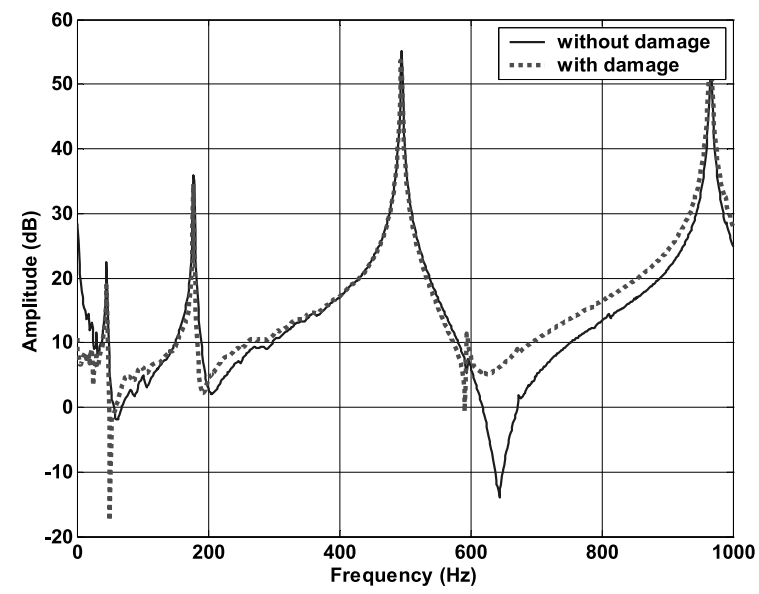

(a) output 1

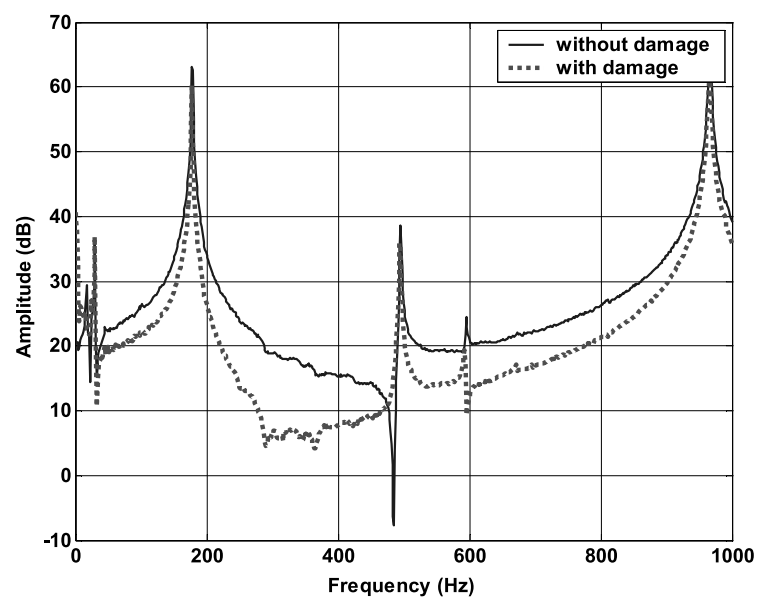

(b) output 2

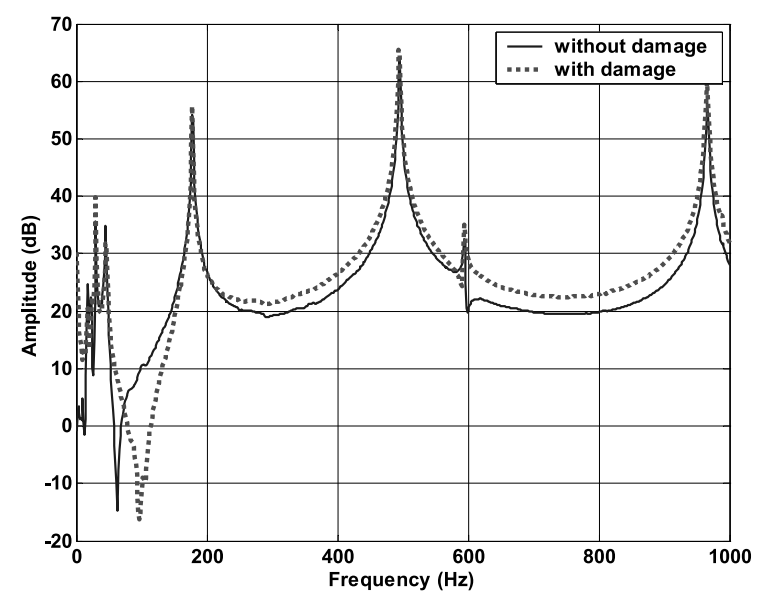

(c) output 3

Fig. 6. Experimental Frequency Response Functions for the healthy and the damaged structure for the set-up shown in Fig. 5 [dB ref $\left.1 \mathrm{~m} / \mathrm{s}^{2} / \mathrm{N}\right]$. 


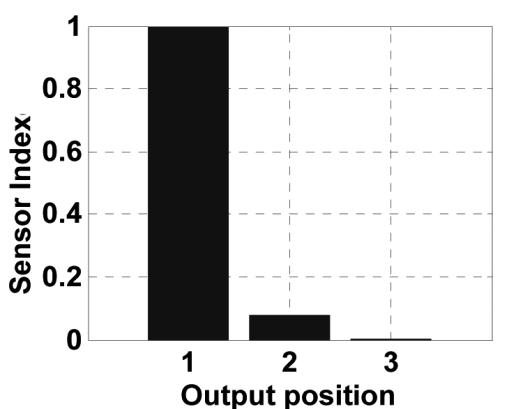

Fig. 7. Sensor index calculated using Eq. (10) for each of the sensor positions - experiments. Damage was simulated by adding 16 g mass at position 1. This should be compared with Fig. $3 \mathrm{a}$, in which, the output position 1 corresponds to sensor position 1 , output position 2 corresponds to sensor position 4 and output position 3 corresponds to sensor position 8 .

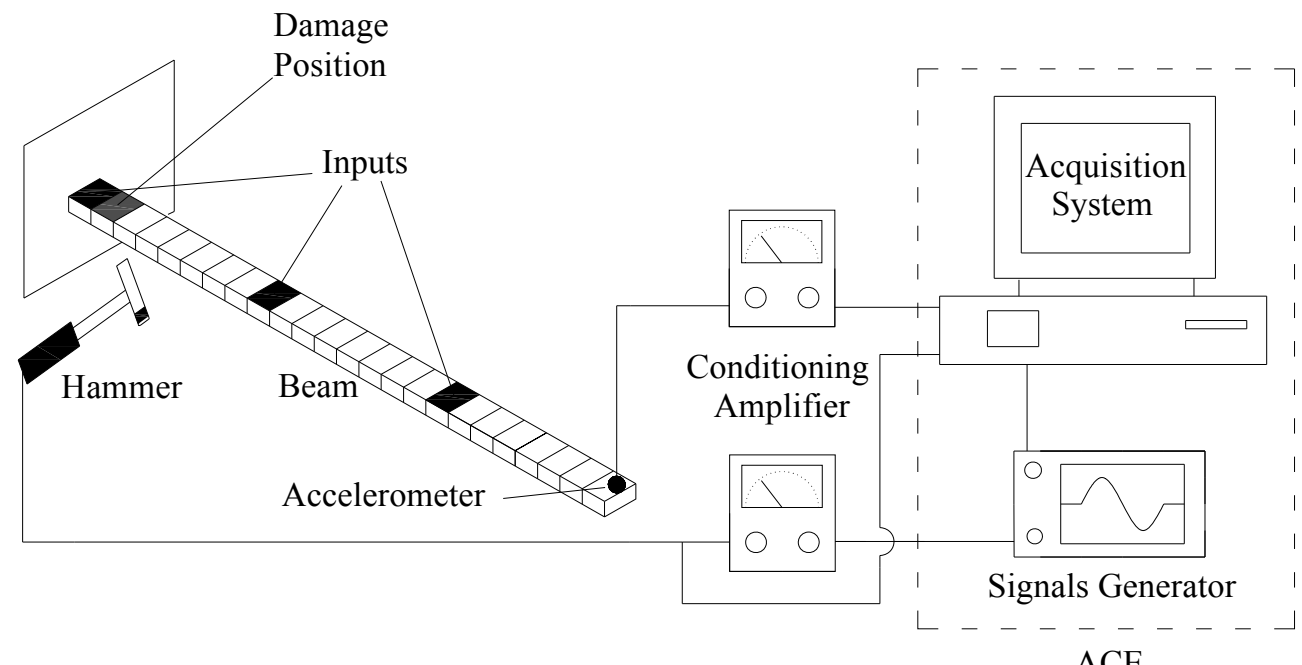

Fig. 8. Experimental setup used for damage location using the controllability grammian.

frequency response functions differ for the structure in the healthy and the damaged states, it is not clear where the damage occurs. Figure 7 shows the sensor index calculated for all three accelerometers using Eq. (10). It can be seen that the damage location was correctly identified.

Figure 8 shows the experimental setup to detect and locate damage using the controlability grammian. As before, damage was simulated by adding additional mass $(16 \mathrm{~g})$ at the same location on the beam. Figures $9 \mathrm{a}-\mathrm{c}$ show the frequency response functions before and after the damage was introduced. Again it is not clear where the damage is located. Using the grammian input index for all three excitation positions given by Eq. (14) the damage location was again correctly identified as can be seen in Fig. 10.

In both cases discussed above, it is clear from the FRFs that the dynamic behavior of the structure has changed as a result of the damage, but it is not clear at all where the damage has occurred. Moreover it can be seen that the frequency response functions measured in the second experiment, when the controllability grammian was used, the FRFs only change by a small amount. It is clear, however, that both grammians prove to be effective metrics, in the cases studied at least, in that they clearly show that the damage has a profound effect on one sensor location or one actuator location depending on the method used. The results of the second method are particularly interesting because they demonstrate a situation which may occur in a more realistic structure where only one sensor can be fitted, and this can be quite remote from the damage. Using an instrumented hammer a quick check can be made on the relative health of the structure easily. 


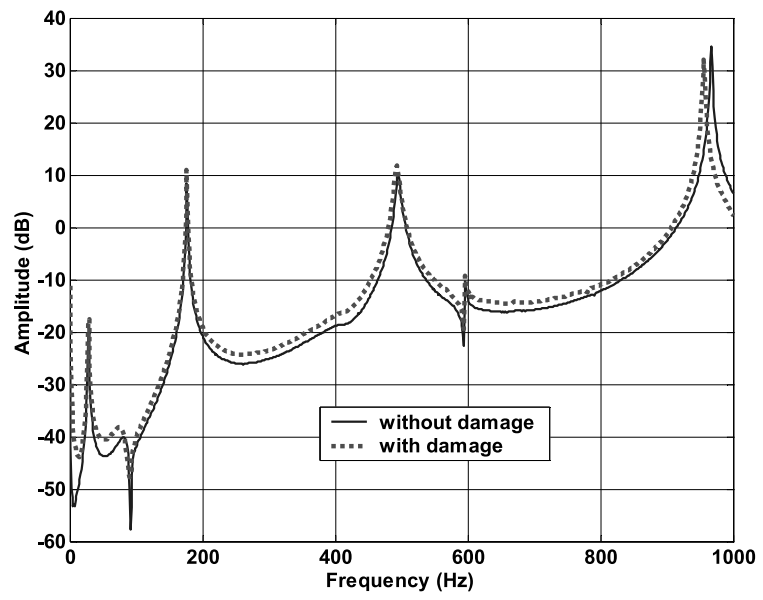

(a) input 1

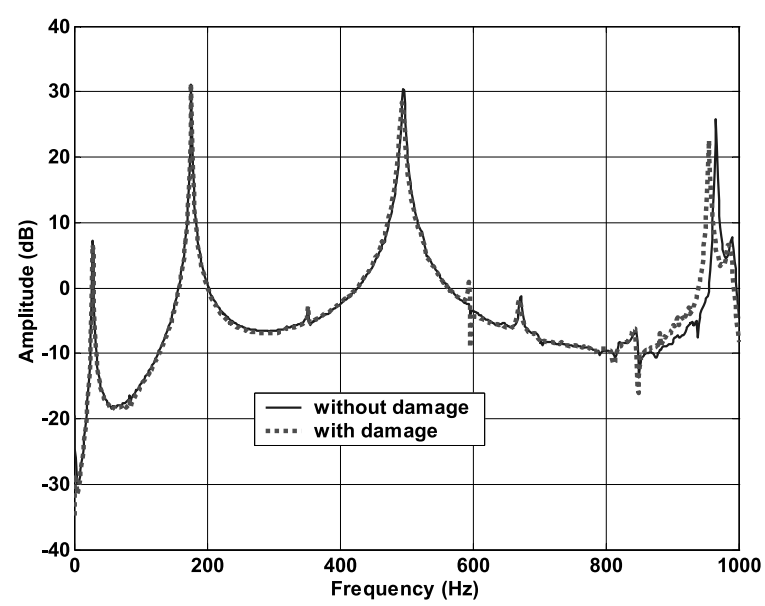

(b) input 2

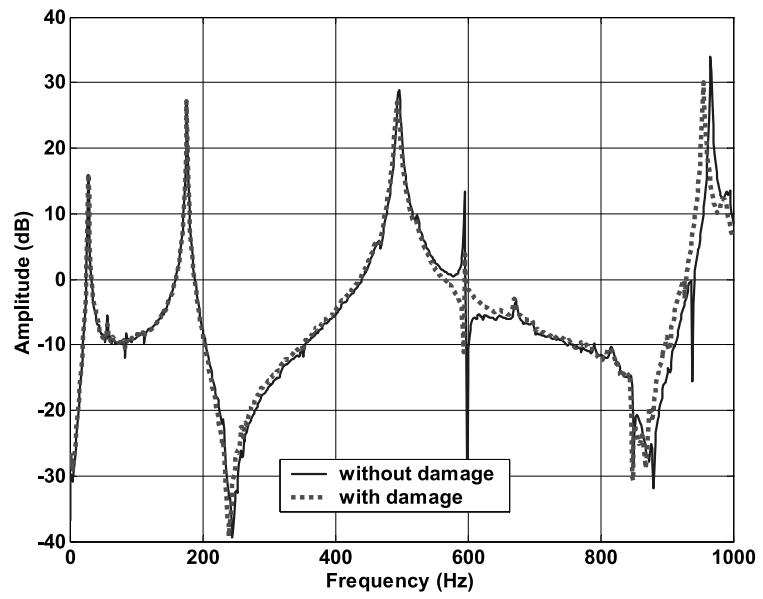

(c) input 3

Fig. 9. Experimental Frequency Response Functions for the healthy and the damaged structure for the set-up shown in Fig. $8\left[\mathrm{~dB}\right.$ ref $\left.1 \mathrm{~m} / \mathrm{s}^{2} / \mathrm{N}\right]$. 


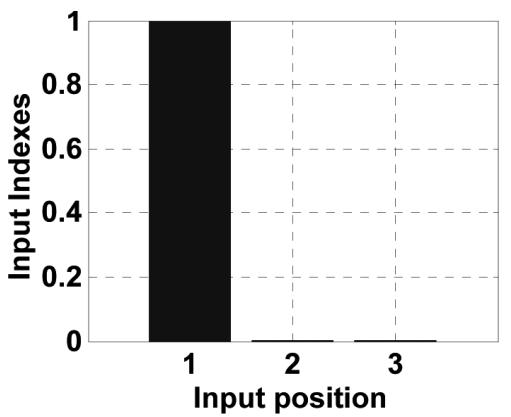

Fig. 10. Input index calculated using Eq. (14) for each of the excitation positions - experiments. Damage was simulated by adding $16 \mathrm{~g}$ mass at position 1. This should be compared with Fig. 4a, in which, the input position 1 corresponds to actuator position 1 , input position 2 corresponds to actuator position 4 and input position 3 corresponds to actuator position 7 .

\section{Conclusions}

This paper has investigated the use of observability and controlability grammian matrices to detect and locate damage in a structure. These matrices have been used extensively in control design and for optimal placement of sensors and actuators in smart structures. In the context of damage detection and location discussed here, these grammians have been used as an indicator of whether damage affects the measured states of the healthy structure. Simulations and experimental results have shown that this approach can be used to give an indication of the damage location. In practical situations, the number of sensors may be limited, so the controllability grammian approach may be preferable as it requires a smaller number of sensing points compared to the observability grammian approach. Simple laboratory scale experiments on a cantilever beam were performed to demonstrate the approach.

\section{Acknowledgments}

The authors acknowledge the support of the Research Foundation of the State of São Paulo (FAPESP-Brazil) and they are also thankful to CNPq and FAPEMIG for partially funding the present research work through the INCT-EIE.

\section{Appendix}

\section{Modal state-space model}

In this appendix, the way in which a state-space model of a structure can be derived from a finite element model is outlined. A finite element model of a structure is given by [8]

$$
\begin{aligned}
& \mathbf{M} \ddot{\mathbf{q}}(t)+\mathbf{D} \dot{\mathbf{q}}(t)+\mathbf{K q}(t)=\mathbf{B}_{0} \mathbf{u}(t) \\
& \mathbf{y}(t)=\mathbf{C}_{\mathbf{q}} \mathbf{q}(t)+\mathbf{C}_{\dot{\mathbf{q}}} \dot{\mathbf{q}}(t)+\mathbf{C}_{\ddot{\mathbf{q}}} \ddot{\mathbf{q}}
\end{aligned}
$$

where $\mathbf{M}, \mathbf{D}$ and $\mathbf{K}$ are the $n \times n$ mass, damping and stiffness matrices respectively; $\mathbf{q}(t)$ is the $n$-length displacement vector and the over dot denotes differentiation with respect to time; the $n \times s$ matrix $\mathbf{B}_{0}$ is the spatial coupling matrix related to the $s$-length input vector $\mathbf{u}(t)$. The $r$-length output vector is $\mathbf{y}(t)$ which is related to the displacement, velocity and acceleration vectors through the matrices given by $\mathbf{C}_{\mathbf{q}}, \mathbf{C}_{\dot{\mathbf{q}}}$ and $\mathbf{C}_{\ddot{\mathbf{q}}}$ respectively. Provided that the damping matrix in equation (A1a) can be diagonalised, Eqs (A1a,b) can be written in modal form as

$$
\begin{aligned}
& \ddot{\mathbf{q}}_{m}(t)+2 \mathbf{Z} \Omega \dot{\mathbf{q}}_{m}(t)+\Omega^{2} \mathbf{q}_{m}(t)=\mathbf{B}_{m} \mathbf{u}(t) \\
& \mathbf{y}(t)=\mathbf{C}_{\mathbf{q} m} \mathbf{q}_{m}(t)+\mathbf{C}_{\dot{\mathbf{q}} m} \dot{\mathbf{q}}_{m}(t)+\mathbf{C}_{\ddot{\mathbf{q}} m} \ddot{\mathbf{q}}_{m i}
\end{aligned}
$$


where $\mathbf{q}_{m}(t)=\Phi^{-1} \mathbf{q}(t)$ and $\Phi$ is the modal matrix; the $n \times n$ damping matrix is given by $\mathbf{Z}=$ $0.5\left(\Phi^{T} \mathbf{M} \Phi\right)^{-1 / 2}\left(\Phi^{T} \mathbf{K} \Phi\right)^{-1 / 2}\left(\Phi^{T} \mathbf{D} \Phi\right)$, the $n \times n$ natural frequency matrix by $\Omega=\left(\Phi^{T} \mathbf{M} \Phi\right)^{-1 / 2}\left(\Phi^{T} \mathbf{K} \Phi\right)^{1 / 2}$, the $n \times s$ modal spatial coupling matrix by $\mathbf{B}_{m}=\left(\Phi^{T} \mathbf{M} \Phi\right)^{-1} \Phi^{T} \mathbf{B}_{0}$, the $r \times n$ modal displacement, velocity and acceleration matrices are given by $\mathbf{C}_{\mathbf{q} m}=\mathbf{C}_{\mathbf{q}} \Phi, \mathbf{C}_{\dot{\mathbf{q}} m}=\mathbf{C}_{\dot{\mathbf{q}}} \Phi$ and $\mathbf{C}_{\ddot{\mathbf{q}} m}=\mathbf{C}_{\ddot{\mathbf{q}}} \Phi$ respectively. Equations (A2a,b) represent a set of $n$ independent equations for each mode of displacement. The equations for the $i$-th mode are given by

$$
\begin{aligned}
& \ddot{q}_{m i}+2 \zeta_{i} \omega_{i} \dot{q}_{m i}+\omega_{i}^{2} q_{m i}=\mathbf{B}_{m i} \mathbf{u} \\
& \mathbf{y}_{i}=\mathbf{C}_{\mathbf{q} m i} q_{m i}+\mathbf{C}_{\dot{\mathbf{q}}_{m i}} \dot{q}_{m i}+\mathbf{C}_{\ddot{\mathbf{q}} m i} \ddot{q}_{m i}
\end{aligned}
$$

where $\mathbf{B}_{m i}$ is the $i$-th row of the modal spatial coupling matrix, and $\mathbf{C}_{q m i}, \mathbf{C}_{\dot{q} m i}$ and $\mathbf{C}_{\ddot{q} m i}$ are the $i$ th columns of the displacement, velocity and acceleration matrices respectively. The modal model, consisting of $n$ modes, can be written in modal state-space form as,

$$
\begin{aligned}
\dot{\mathbf{x}}(t) & =\mathbf{A} \mathbf{x}(t)+\mathbf{B u}(t) \\
\mathbf{y}(t) & =\mathbf{C} \mathbf{x}(t)
\end{aligned}
$$

where the state vector, $\mathbf{x}$, in modal coordinates consists of $2 n$ independent components, $\mathbf{x}_{i}$, that represents the state of each mode. The $i$-th modal state component is given by $\mathbf{x}_{i}=\left\{\begin{array}{l}q_{m i} \\ q_{m o i}\end{array}\right\}$, where $q_{m o i}=\zeta_{i} q_{m i}+\frac{\dot{q}_{m i}}{\omega_{i}}$. In Eq. (A4a) the system matrix is given by the block diagonal matrix $\mathbf{A}=\operatorname{diag}\left(\mathbf{A}_{i}\right)$ where

$$
\mathbf{A}_{i}=\left[\begin{array}{ll}
-\zeta_{i} \omega_{i} & \omega_{i} \\
\omega_{i}\left(\zeta_{i}^{2}-1\right) & -\zeta_{i} \omega_{i}
\end{array}\right]
$$

is a $2 \times 2$ block, and the spatial coupling matrix is given by the block matrix

$$
\mathbf{B}=\left[\begin{array}{l}
\mathbf{B}_{1} \\
\mathbf{B}_{i} \\
\vdots \\
\mathbf{B}_{n}
\end{array}\right]
$$

where $\mathbf{B}_{i}=\left[\begin{array}{l}\mathbf{0} \\ \mathbf{B}_{m i}\end{array}\right]$ is a $2 \times s$ block. In Eq. (A4b), $\mathbf{C}=\left[\mathbf{C}_{1} \mathbf{C}_{i} \cdots \mathbf{C}_{n}\right]$ is a block matrix, where $\mathbf{C}_{i}=\left[\mathbf{C}_{1 i} \mathbf{C}_{2 i}\right]$ is an $r \times 2$ block, and $\mathbf{C}_{1 i}=\mathbf{C}_{\mathbf{q} m i}-\omega_{i} \mathbf{C}_{\dot{\mathbf{q}} m i}+\omega_{i}^{2}\left(2 \zeta_{i}^{2}-1\right) \mathbf{C}_{\ddot{\mathbf{q}} m i}$ and $\mathbf{C}_{2 i}=\omega_{i}\left(\mathbf{C}_{\dot{\mathbf{q}} m i}-2 \zeta_{i} \omega_{i} \mathbf{C}_{\ddot{\mathbf{q}} m i}\right)$.

\section{References}

[1] A. Arbel, Controllability Measures and Actuator Placement in Oscillatory Systems, International Journal of Control 33(3) (1981), 565-574.

[2] I. Bruant and L. e Proslier, Optimal Location of Actuators and Sensors in Active Vibration Control, Journal of Intelligent Material Systems and Structures 16 (2005), 197-206.

[3] D.D. Bueno, C.R. Marqui, Jr., V. Lopes and M.J. Brennan, Structural Damage Location Using Observability Grammian Matrices, Ninth International Conference on Recent Advances in Structural Dynamics, 2006, Southampton, RASD2006, 2006.

[4] A. Deraemaeker and A. Preumont, Vibration based Damage detection using Large Array Sensors and Spatial Filters, Mechanical Systems and Signals Processing 20 (2006), 1615-1630.

[5] G.B. Daniel, T.S. Morais and G.P. Melo, Technique of Parameters and Inputs Identification in Mechanical Systems, 18th International Congress of Mechanical Engineering, Ouro Preto, MG, Brazil, 2005.

[6] C.R. Farrar, H. Sohn, F.M. Hemez, M.C. Anderson, M.T. Bement, P.J. Cornwell, S.W. Doebling, J.F. Schultze, N. Lieven and A.N. Robertson, Damage Prognosis: Current Status and Future Needs, Los Alamos National Laboratory Report, LA-14051-MS, 2004.

[7] M.I. Friswell and J.E. Penny, The Practical Limits of Damage Detection and Location using Vibration Data, Proceedings, 11th VPI\&SU Symposium on Structural Dynamics and Control, Blacksburg, VA, 1-10, 1997.

[8] W. Gawronski, Dynamics and Control of Structures, A Modal Approach, 1. Ed. New York, Springer Verlag, 1998.

[9] W. Gawronski and J.T. Sawicki, Structural Damage Detection Using Modal Norms, Journal of Sound and Vibration 229(1) (2000), 194-198. 
[10] A. Hac and L. Liu, Sensors and Actuator Location in Motion Control of Flexible Structures, Journal of Sound and Vibration 167(2) (1993), 239-261.

[11] S.R. Hall and T.J. Conquest, The Total Data Integrity Initiative - Structural Health Monitoring, The Next Generation, (2nd ed.), Proceedings of the USAF ASIP, 1999.

[12] A. Heniche, I. Kamwa and R. Grondin, Torsional-Mode Identification Turbogenerators with Application to PSS Tuning, International Conference on Power Systems Transients, in Montreal, Canada on June 19-23, paper N ${ }^{\circ}$ IPST05 — $222,2005$.

[13] J.N. Juang, Applied System Identification, Prentice Hall, Englewood Hills, NJ, 1994.

[14] J. Juang and M.Q. Phan, Identification and Control of Mechanical Systems, Cambridge University Press, ISBN 0521783550, 2001.

[15] T. Kailath, Linear Systems, Prentice Hall, Englewood Cliffs, NJ, 1980.

[16] R.E. Kalman, Y.C. Ho and K.S. Narenda, Controllability of Linear Dynamics System, Contribution to Differential Equations 1(2) (1962), 189-213.

[17] S. Leleu, Abou-Kandil and Y. Bonnassieux, Piezoelectric Actuators and Sensors Location for Active Control of Piezoelectric Structures, IEEE Transactions on Instrumentation and Measurement 50(6) (2001).

[18] K.B. Lim, Method for Optimal Actuator and Sensor Placement for Large Flexible Structures, Journal of Guidance 15(1) (1992), 49-57.

[19] N. Maia, J. Silva et al., Theoretical and Experimental Modal Analysis, Research Studies Press Ltd., Baldock, Hertfordshire, England.

[20] C. Marantidis, C.B. Van Way and J.N. Kudva, Acoustic-Emission Sensing in an On-Board Smart Structural Health Monitoring System for Military Aircraft, Proceedings of the SPIE Conference on Smart Structures and Integrated Systems 2191 (1994), 258-264

[21] C.R. Marqui, D.D. Bueno, L. Cordeiro, C.R. Silva and V. Lopes, Jr., Experimental Damage Detection Using System Norms, XXIV IMAC International Modal Analysis Conference, St. Louis, Missouri, USA, 2006.

[22] A. Mita, Emerging Needs in Japan for Health Monitoring Technologies in Civil and Building Structures, Proc. Second International Workshop on Structural Health Monitoring, Stanford University, 1999, pp. 56-67.

[23] R.P. Pacheco and V. Steffen, Jr., On the identification of non-linear mechanical systems using orthogonal functions, International Journal of Non-linear Mechanics, Oxford, UK 39(7) (2004), 1147-1159.

[24] F. Peng, A. Ng and Y.Y. e Hu, Actuator Placement Optimization and Adaptive Vibration Control of Plate Smart Structures, Journal of Intelligent Material Systems and Structures 6 (2005), 263-271.

[25] G. Schulz and G. Heimbold, Dislocated Actuator/Sensor Positioning and Feedback Design for Flexible Structures, Journal of Guidance 6(5) (1983), 361-367.

[26] G. Silva, M. Dias Júnior and V. Lopes, Jr., Structural Health Monitoring in Smart Structures Through Time Series Analysis, Structural Health Monitoring 7(3) (2008), SAGE Publications, DOI: 10.1177/1475921708090561, 231-244.

[27] G. Sohn, G. Farrar, F.M. Hemez, D.D. Shunk, S.W. Stinemates, B.R. Nadler and J.J. Czarnecki, A Review of Structural Health Monitoring Literature form 1996-2001, Los Alamos National Laboratory report LA-13976-MS (2004).

[28] H. Van der Auweraer and H. Peeters, International Research Projects on Structural Health Monitoring: An Overview, Structural Health MonitoringJournal, Dec 2003; vol. 2: pp. 341-358.

[29] H. Zhou, 1995, Robust and Optimal Control, Prentice Hall, Englewood Hills, NJ. 

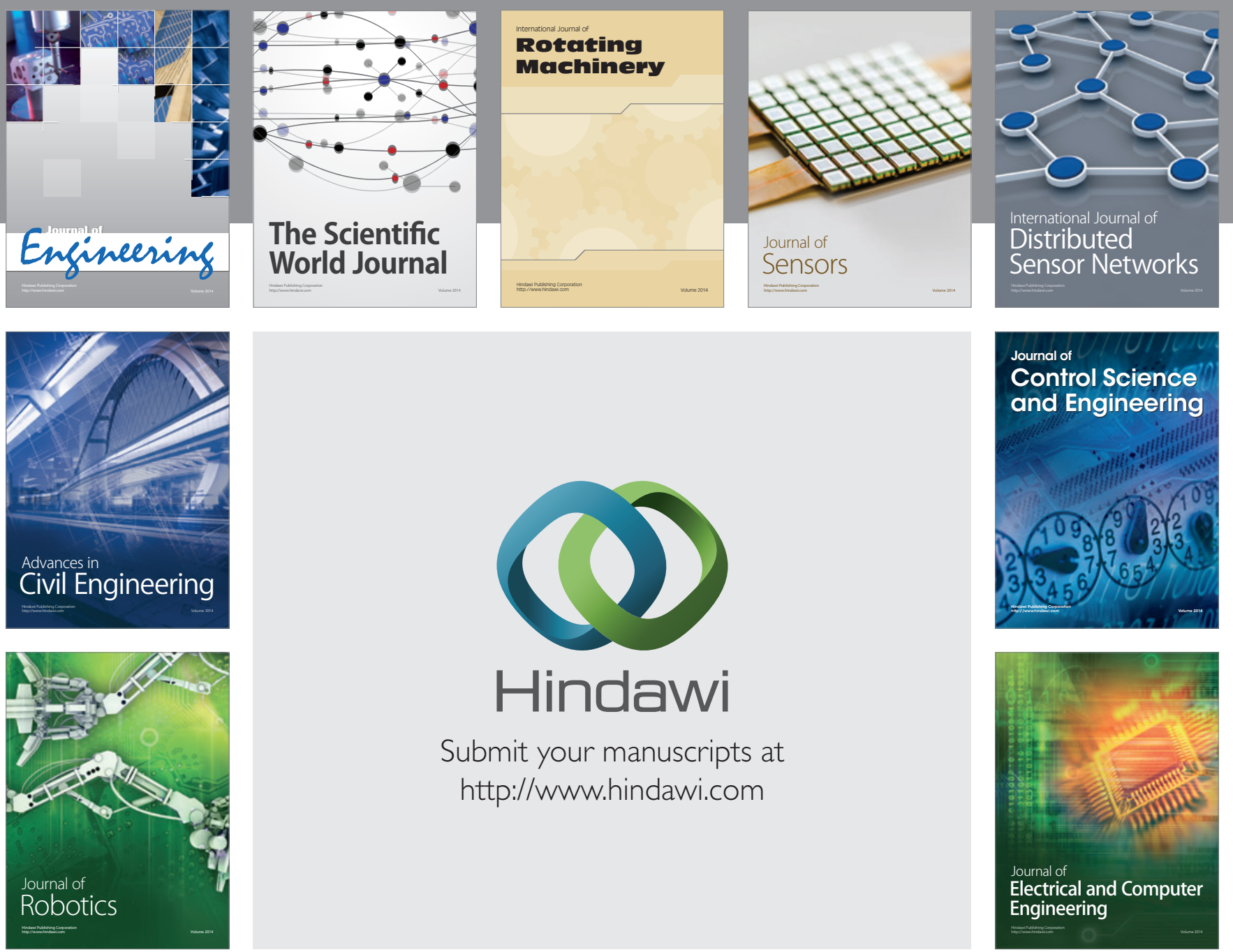

Submit your manuscripts at

http://www.hindawi.com
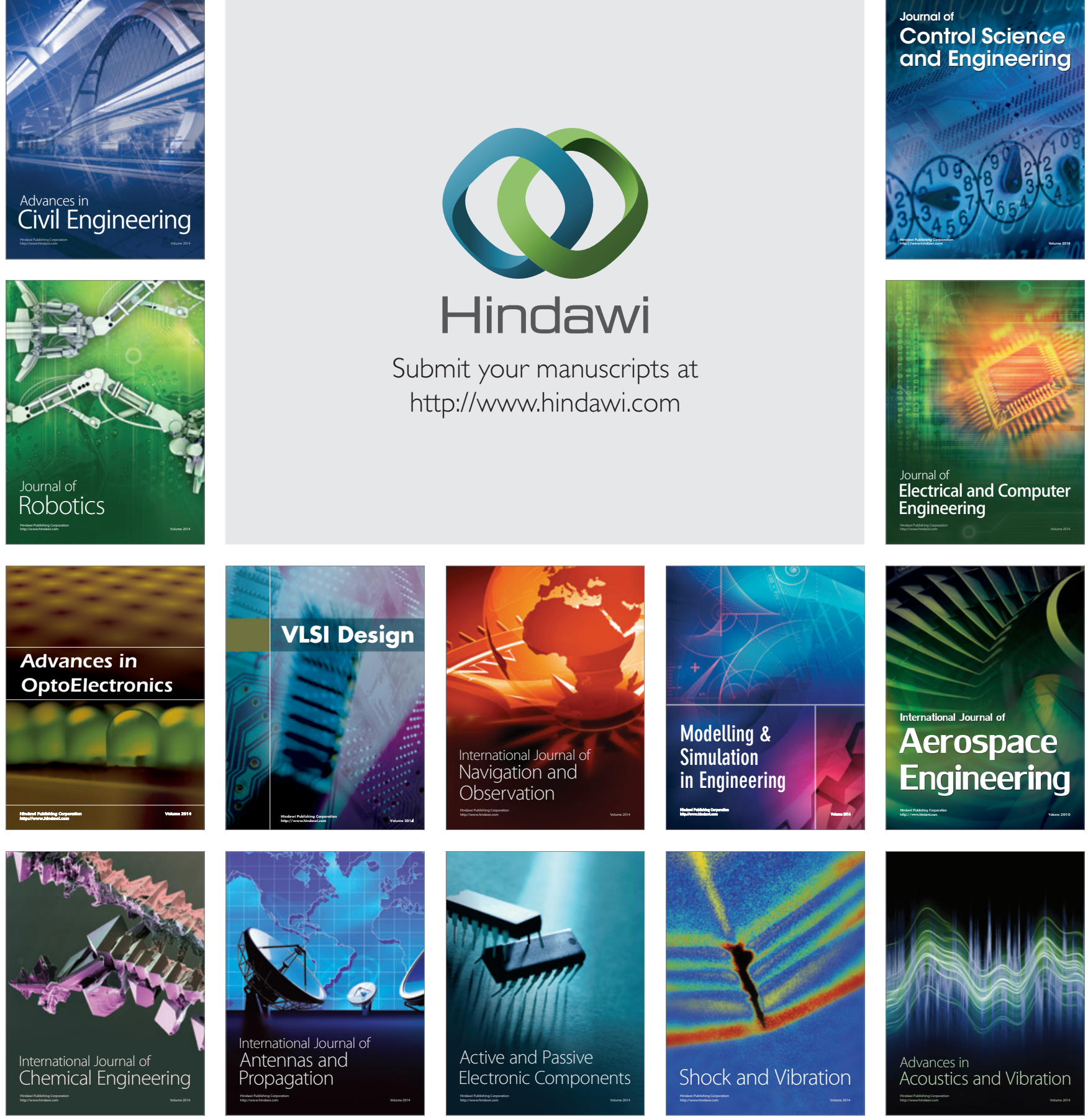